\section{Políticas de saúde no Brasil nos anos 2000: a agenda federal de prioridades}

\author{
Health policies in Brazil in the 2000s: \\ the national priority agenda
}

\author{
${ }^{1}$ Escola Nacional de Saúde \\ Pública Sergio Arouca \\ Fundação Oswaldo Cruz, Rio \\ de Janeiro, Brasil. \\ 2 Museu Nacional, \\ Universidade Federal do Rio \\ de Janeiro, Rio de Janeiro, \\ Brasil. \\ Correspondência \\ C. V. Machado \\ Departamento de \\ Administração e Planejamento \\ em Saúde, Escola Nacional de \\ Saúde Pública Sergio Arouca, \\ Fundação Oswaldo Cruz. \\ Rua Leopoldo Bulhões 1480, \\ Rio de Janeiro, RJ 21041-210, \\ Brasil. \\ cristiani@ensp.fiocruz.br
}

\section{Abstract}

This article analyzes Brazilian national health priorities from 2003 to 2008 under the Lula Administration. The study included a literature review, document analysis, and interviews with Federal health administrators. Four priorities were identified on the national health agenda: the Family Health Program, Smiling Brazil, Mobile Emergency Services, and the Popular Pharmacy Program. The first is a policy with high institutional density launched by the previous Administration, constituting an example of path dependence. The other three are innovations in areas where there had been weaknesses in Federal government action. The four policy priorities are strategies focused on solving key problems in the Brazilian health system. However, they display important differences in their historical development, political and institutional base, inclusion on the Federal agenda, and implications for the principles of the Unified National Health System. Although incremental changes have been introduced, national health policy has been characterized predominantly by continuity.

Health Policy; Health Priority Agenda; Single Health System; Federal Government

\author{
Cristiani Vieira Machado 1 \\ Tatiana Wargas de Faria Baptista 1 \\ Carolina de Oliveira Nogueira 2
}

\section{Introdução}

A história das políticas de saúde no Brasil, particularmente sua relação com a construção das bases do Estado Nacional e com as características do modelo de intervenção estatal na área econômica e social conformado ao longo do século XX 1, impõe dificuldades à delimitação do estudo dessa política setorial em um governo específico.

Este artigo analisa as políticas de saúde no período recente, com foco nas prioridades da agenda federal durante o Governo Lula. Buscase, entretanto, considerar elementos da trajetória anterior do sistema de saúde. Afinal, qual o legado da política de saúde quando Lula assume em 2003? Quais as possibilidades do novo governo para a viabilização de transformações na realidade sanitária no país?

Um ponto de partida importante para a análise diz respeito à compreensão das dificuldades de consolidação de um sistema público e universal de saúde no Brasil. Na década de 1980, a reforma sanitária brasileira partiu de críticas ao caráter fragmentado, privatista e excludente do sistema de saúde até então 2,3,4. Houve experiências relevantes de reorganização setorial com expansão assistencial, como as Ações Integradas de Saúde e o Sistema Único Descentralizado de Saúde 4,5. Na Constituição Federal de 1988, a saúde foi reconhecida como direito de cidadania e inserida em uma concepção ampla de Segurida- 
de Social, a ser garantida mediante políticas econômicas e sociais abrangentes e pela instituição do Sistema Único de Saúde (SUS).

A implantação do projeto da reforma sanitária exigia o enfrentamento de distorções estruturais do sistema de saúde e tinha como desafio a superação do grave quadro de desigualdades em saúde no país. Isso seria viável no bojo de um projeto amplo de transformação do modelo de desenvolvimento e da sociedade brasileira, que aliasse crescimento econômico, fortalecimento dos valores democráticos e avanços sociais.

No entanto, parte das conquistas constitucionais foi bloqueada pelas consequências da inflexão conservadora do final dos anos 1980, que culminou na eleição de Collor para a Presidência da República em 1989. A década de 1990 no Brasil foi marcada pela convivência entre democratização e liberalização econômica ${ }^{6}$.

O percurso da política de saúde nos anos 1990 expressou tensões entre a agenda da reforma sanitária e a agenda de reforma do Estado de inspiração liberal 7,8,9,10. Mesmo em um contexto adverso, houve avanços político-institucionais como a unificação do comando da política nacional de saúde no âmbito do Ministério da Saúde e a construção de um arcabouço institucional e decisório para o SUS que respeitasse a lógica federativa e a diretriz de participação social. Outro avanço se refere à expansão das ações e serviços públicos de saúde no território nacional ${ }^{11}$.

Contudo, a implementação do SUS foi prejudicada pelo processo de reforma do Estado hegemônico naquela década, contrário à lógica de proteção social abrangente. Algumas evidências disso foram: as dificuldades na consolidação do modelo de Seguridade Social, tais como a evolução fragmentada das políticas sociais com baixa integração 12 e as restrições ao financiamento das políticas sociais, incluindo a saúde 13,14; as condições adversas em que ocorreu o processo de descentralização em saúde, relacionadas ao ajuste fiscal, às restrições à contratação de pessoal e à heterogeneidade dos municípios brasileiros 15,16,17; os problemas no campo da gestão do trabalho e da formação de profissionais de saúde 18; as deficiências na produção e distribuição nacional de insumos relevantes para a saúde (medicamentos, fármacos, vacinas e equipamentos), com agravamento da situação de dependência tecnológica que ameaça a sustentabilidade do SUS em médio e longo prazo 19; a persistência de problemas nas relações público-privadas em saúde, como a dependência do SUS aos prestadores privados e o peso do segmento de planos e seguros de saúde 20,21 .

Ao final da década de 1990, a saúde registrava uma situação paradoxal, verificada em outras áreas sociais, em que avanços institucionais e em programas específicos conviviam com a persistência de graves desigualdades sociais 22 .

Outro aspecto a ser considerado na análise da política de saúde é o modelo do federalismo brasileiro, que tem implicações para a definição do papel das esferas de governo na saúde. A descentralização no setor nas últimas duas décadas, com ênfase nos municípios, alterou o papel federal na política. Porém, mesmo em um contexto de pressões por retração do Estado e de descentralização político-administrativa, reafirmou-se a relevância do Ministério da Saúde na definição de políticas, no financiamento e na regulação do sistema de saúde 23,24. Isso parece ter relação com características estruturais do Estado brasileiro, historicamente marcado pelo peso do Executivo federal. Ademais, é consoante com a tendência de fortalecimento do papel de regulação dos ministérios da saúde em vários países nos últimos anos, apesar das pressões por retração dos Estados Nacionais 25 .

O início do Governo Lula trouxe expectativas quanto à inflexão no modelo de desenvolvimento e de proteção social. Entre 2003 e 2008, houve destaque governamental para as políticas de redução da pobreza, o que contribuiu para a redução da desigualdade de renda no país, aferida pelo coeficiente de Gini 26 . Em termos do gasto social federal, as duas áreas que contaram com maior incremento foram a assistência social (em termos relativos) e a previdência (em valores absolutos). Já os gastos com educação e saúde têm apresentado oscilações em termos de participação no Produto Interno Bruto. Ainda assim, a saúde representa o segundo maior orçamento da área social. Entretanto, além dos estudos quantitativos, é importante analisar qualitativamente a condução governamental de políticas universais como a de saúde.

Ainda existem poucos estudos sobre as políticas de saúde no Governo Lula 27,28. Este artigo discute as continuidades e mudanças nas políticas setoriais entre 2003 e 2008, com foco na análise das prioridades da agenda governamental. Assim, visa contribuir para uma melhor compreensão das possibilidades e limites da atuação federal no que tange à superação dos problemas do sistema nacional de saúde.

\section{Metodologia}

A pesquisa se baseou no enfoque do institucionalismo histórico, que destaca dois aspectos no estudo das políticas: (a) o contexto das instituições, definidas como regras socialmente construídas que estruturam condutas; (b) a di- 
mensão temporal. Tal abordagem também reconhece o papel dos atores nas políticas públicas, ressaltando, no entanto, que suas preferências e decisões são condicionadas pelas instituições vigentes 29 .

Valorizou-se a concepção de dependência da trajetória ("path-dependence"), segundo a qual decisões precedentes em uma dada direção induzem movimentos subsequentes na mesma direção. Pierson 30 conceitua "path dependen$c e$ " como um processo social ancorado em uma dinâmica de retornos crescentes ou de retroalimentação positiva. Dessa forma, procurou-se analisar se a definição de políticas federais prioritárias no período estudado foi influenciada por escolhas em momentos anteriores da trajetória da política (elementos de continuidade) e/ou expressou movimentos de alterações nas regras vigentes (elementos de mudança).

O estudo trata da fase de formulação de políticas, com destaque para a conformação da agenda governamental. Para Kingdon 31, agenda é a relação de temas ou problemas que mobilizam seriamente a atenção de governantes e pessoas a eles associadas em determinado momento. Nesse sentido, o estudo procurou identificar e caracterizar as políticas que tiveram destaque na agenda federal da saúde no período.

As seguintes estratégias metodológicas foram adotadas:

- Análise de documentos oficiais selecionados por sua relevância na explicitação das prioridades do governo e do Ministério da Saúde: programas de governo (Coligação Lula Presidente. Saúde para a Família Brasileira; 2002/Coligação A Força do Povo. Programa Setorial de Saúde 2007/2010; 2006), planos nacionais de saúde 32,33, entre outros;

- Análise de documentos 34,35,36,37 e portarias referentes às políticas prioritárias, obtidas do Sistema de Legislação da Saúde do Ministério da Saúde (Saúde Legis);

- Análise de dados primários fornecidos pelo Ministério da Saúde e de dados secundários dos sistemas de informações oficiais;

- Análise orçamentária federal, com base em dados do Sistema de Informações sobre Planos e Orçamento Público (SIGA) ou disponíveis na Sala de Situação do Ministério;

- Realização de 23 entrevistas semiestruturadas com dirigentes e técnicos federais atuantes no período, incluindo dois ex-ministros, secretários de primeiro escalão e coordenadores de áreas e políticas estratégicas.

Para a identificação das prioridades federais da saúde, foram considerados como critérios: o destaque nos documentos oficiais e no discurso dos dirigentes entrevistados; dados de implanta- ção das políticas (evolução quantitativa e distribuição territorial); a evolução dos gastos federais por políticas; a capacidade das políticas gerarem inovações específicas (nas regras de financiamento, organização ou provisão de serviços de saúde) e/ou demandas para as diversas áreas do Ministério da Saúde (por exemplo, relativas à formação de profissionais).

As políticas que preencheram esses critérios foram analisadas mediante as seguintes categorias: origens e trajetória da política; motivos e atores que influenciaram o seu destaque na agenda federal; implantação nacional (quantidade de serviços/equipes, abrangência populacional estimada); implicações em termos de continuidade ou mudança nas políticas nacionais de saúde.

Quanto à abrangência populacional, foram consideradas estimativas oficiais do Ministério da Saúde, que utilizam os dados populacionais do Instituto Brasileiro de Geografia e Estatística e seguem os seguintes parâmetros: cada equipe de saúde da família abrange 3.450 pessoas e cada equipe de saúde bucal abrange 6.900 pessoas (até o limite populacional do município); cada Serviço de Atendimento Móvel de Urgência (SAMU) abrange toda a população da área definida na ocasião de sua implantação.

A investigação foi aprovada por Comitê de Ética em Pesquisa e seguiu as normas relativas a pesquisas com seres humanos.

\section{Resultados}

A análise das prioridades na agenda federal da saúde mostra continuidades e mudanças na condução da política setorial no período de 2003 a 2008. Foram identificadas quatro políticas prioritárias:

- Política de Estado de alta densidade institucional (prioridade anterior, que persiste na agenda federal de sucessivas gestões ministeriais): Estratégia Saúde da Família (ESF).

- Políticas priorizadas na agenda do Governo Lula, assumidas como prioridade pelo Ministério da Saúde: Brasil Sorridente, SAMU e Farmácia Popular.

A ESF representa uma estratégia de reorientação do sistema de saúde brasileiro com base na atenção primária, que compreende a conformação de equipes profissionais compostas por médico, enfermeiro, auxiliar de enfermagem e agentes comunitários de saúde. Tais equipes são implantadas tendo em vista diretrizes relacionadas à definição de território e população de abrangência e devem orientar-se por princípios como o estabelecimento de vínculos com os usuários, busca de integração do sistema e coordenação do cuidado. 
A ESF já havia representado uma política prioritária para a organização da atenção primária em saúde no Governo Fernando Henrique Cardoso (1995-2002) 38, com incentivos para a sua implementação. No início dos anos 2000, a estratégia havia alcançado uma importante disseminação no território nacional e uma expressiva base de apoio política, técnica e social 39,40. Quando se inicia o Governo Lula em 2003, a ESF permanece na agenda e prossegue a sua expansão. Ao final de 2008, segundo estimativas oficiais, a ESF abrangeria $49 \%$ da população (Figura 1), o que corresponderia a mais de 90 milhões de pessoas. Essa estimativa é próxima da obtida pela Pesquisa Nacional por Amostras de Domicílio (PNAD) para o mesmo ano 41.

A análise dos documentos oficiais e das entrevistas sugeriu que, no período estudado, a ESF permaneceu como prioridade na agenda federal. A valorização da ESF pelo Ministério da Saúde também foi evidenciada por um aumento expressivo de gastos com o programa e pela sua influência na definição de propostas nos vários campos de atuação em saúde. Por exemplo, as políticas federais relativas à formação de profissionais de saúde no período se voltaram particularmente para a ESF.

As outras três políticas de saúde priorizadas na agenda do Governo Lula - o Brasil Sorridente, o SAMU e a Farmácia Popular - representaram iniciativas de enfrentamento de problemas concretos do sistema de saúde. Todavia, cabe observar que as três políticas têm pontos de partida, trajetórias institucionais e bases de apoio diferentes.

O Brasil Sorridente é uma política de ampliação e qualificação da atenção em saúde bucal em todos os níveis, buscando assegurar inclusive atendimento de maior complexidade. A política preconiza a expansão de equipes de saúde bucal junto às equipes de saúde da família, define como estratégia-chave a implantação de centros de especialidades odontológicas de referência para as equipes de saúde bucal e propõe a implantação de laboratórios de próteses dentárias 35 .

A entrada da saúde bucal na agenda de prioridades do governo efetiva-se dezessete anos após a realização da I Conferência Nacional de Saúde Bucal e do I Levantamento Epidemiológico em Saúde Bucal (ambos de 1986), quando já se denunciava a precária situação de saúde bucal da população e os limites da atenção nessa área. Nos anos 1990, a área manteve baixo prestígio político e apenas em 2001 ocorre uma inovação na trajetória da política, com a implantação das equipes de saúde bucal articuladas às equipes de saúde da família.
Em 2002, no período de transição de governos, abriu-se uma "janela de oportunidade" 31 para a saúde bucal. Havia no país um movimento de profissionais de odontologia articulados à reforma sanitária, que há décadas lutava pelo fortalecimento da Saúde Bucal Coletiva 42 . Os representantes desse movimento foram bem sucedidos em emplacar a saúde bucal como prioridade no Governo Lula e na proposição de um desenho abrangente para a política, envolvendo ações de promoção, prevenção, tratamento e reabilitação dentária 35 .

Diversos entrevistados ressaltaram que a adoção do Brasil Sorridente como marco governamental foi fundamental para permitir mudanças substantivas nessa área, atendendo reivindicações históricas de especialistas, com o propósito de melhorar as condições de saúde bucal da população.

O Brasil Sorridente, em que pese o seu desenho abrangente, avançou mais até o momento no tocante à expansão das equipes de saúde bucal atreladas à ESF do que nos demais componentes. Entre 2002 e 2008, o número de equipes de saúde bucal implantadas no país passou de 4.261 para 17.349 , representando um aumento de $407 \%$. A abrangência populacional estimada dessas equipes aumentou de $15 \%$ para $45 \%$, ultrapassando 85 milhões de pessoas (Figura 1). Quanto aos centros de especialidades odontológicas e aos laboratórios de prótese dentária, foram implantados até 2008, respectivamente, 672 e 321 unidades. Conforme dados do Ministério da Saúde, há unidades implantadas em todos os estados, com expansão gradativa do número de unidades ano a ano.

Outra política que ganhou destaque na agenda da saúde a partir de 2003 foi a implantação dos SAMU, que representam a vertente de atendimento pré-hospitalar móvel da política nacional de atenção às urgências. $\mathrm{O}$ propósito geral da estratégia é melhorar o acesso e a qualidade do atendimento às urgências e emergências médicas no país, compreendendo atendimentos nas áreas clínica, pediátrica, cirúrgica, traumática, gineco-obstétrica e de saúde mental. Cada SAMU é constituído por uma central de regulação médica, uma equipe de profissionais e um conjunto de ambulâncias, de suporte básico ou intensivo, que podem ter abrangência municipal ou regional. O governo federal, além do papel de regulamentação, financia a implantação das centrais, a aquisição das ambulâncias e parte da manutenção dos serviços em todo o território nacional.

As origens da política nacional de urgências são anteriores ao Governo Lula, sendo que em 2002 uma Portaria Federal havia traçado diretri- 
Evolução da abrangência populacional estimada das equipes de saúde da família e das equipes de saúde bucal. Brasil, 1994-2008

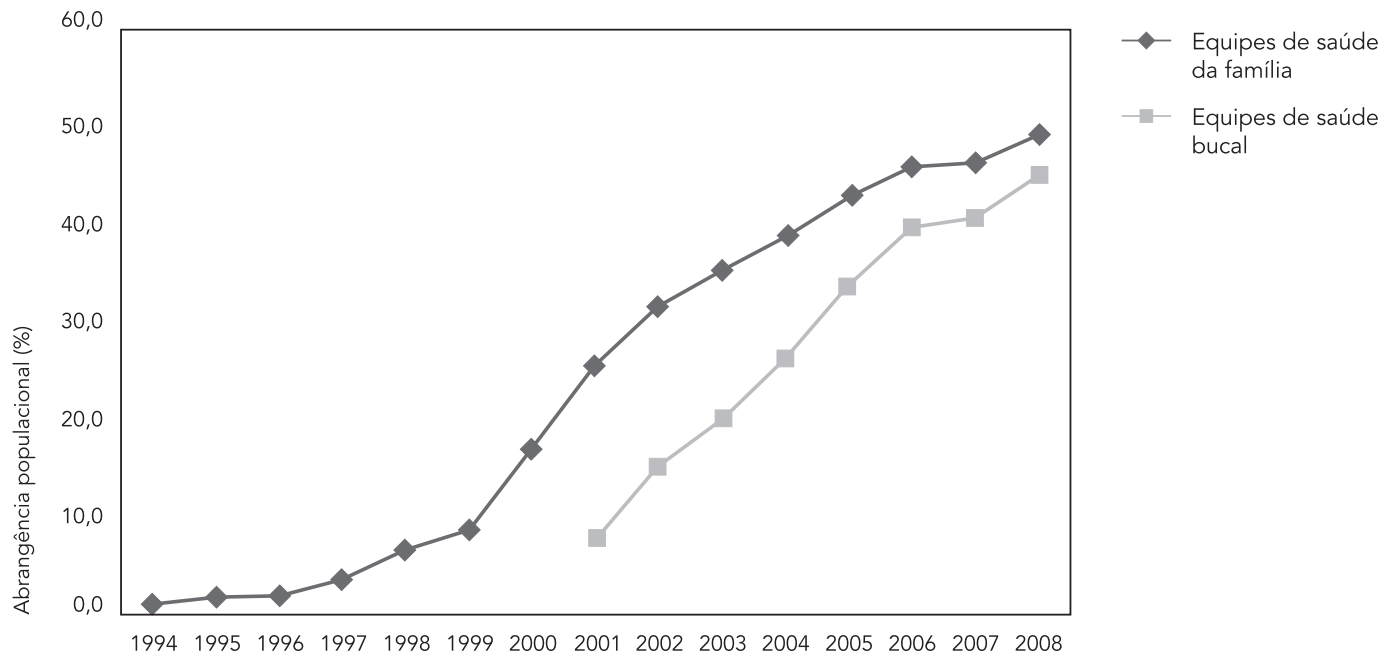

Fonte: dados de abrangência populacional estimada do Departamento de Atenção Básica do Ministério da Saúde.

Dados populacionais do Instituto Brasileiro de Geografia e Estatística.

zes para a organização da atenção às urgências no sistema de saúde. No entanto, seu destaque na agenda federal da saúde e a ênfase no atendimento pré-hospitalar representam novidades, favorecidos pelo apoio do Presidente e do primeiro ministro da Saúde do período. Segundo entrevistados e documentos oficiais 36 , tal estratégia se inspira em modelos internacionais - como o francês e o americano - e em experiências locais bem-sucedidas no país. Entre 2004 e 2008, os SAMU apresentaram uma rápida expansão, alcançando 146 serviços no território nacional em 2009. Segundo dados oficiais, isso corresponde a uma abrangência estimada de mais de 1.200 municípios e de cerca 100 milhões de pessoas, ou seja, mais da metade da população brasileira reside na área de abrangência dos SAMU. Dada a necessidade de adesão de estados e municípios, a abrangência da estratégia é muito variada entre as unidades da federação, o que se traduz em diferenças regionais (Figura 2).

Em que pese a relevância do atendimento pré-hospitalar das urgências para os sistemas públicos de saúde, vários desafios têm sido levantados. Um deles se relaciona à capacidade de atendimento do SAMU, tendo em vista que a maioria das ambulâncias disponíveis é de suporte básico (destinadas a primeiro atendimento por técnico de enfermagem e transporte) e somente 21,5\% são ambulâncias de suporte avançado, que contam com a presença do profissional médico e equipamentos para cuidados intensivos. Ressalte-se ainda necessidade de organização regional de uma rede de serviços de saúde integrada e preparada para esse tipo de atendimento, sem a qual a estratégia do SAMU pode ter alcance limitado. Recentemente, houve iniciativas federais de ampliação do acesso ao atendimento das urgências, por meio da expansão de unidades de pronto atendimento e de reformas de hospitais de emergência, valorizadas nas entrevistas e na regulamentação nacional recente.

A quarta política de saúde priorizada no Governo Lula é o programa Farmácia Popular, lançado como proposta na campanha eleitoral para a Presidência de 2002. Tal política se volta para o aumento do acesso das pessoas a medicamentos a baixos preços para as famílias, com subsídio do Governo Federal, configurando uma estratégia de copagamento entre usuários e Estado.

O documento de diretrizes do programa 37 refere os altos gastos das famílias brasileiras com medicamentos e aponta como população-alvo preferencial as pessoas que têm dificuldades de adquiri-los após atendimento em serviços de 
Evolução da abrangência populacional estimada dos Serviços de Atendimento Móvel de Urgência (SAMU), por região. Brasil, 2004-2008.

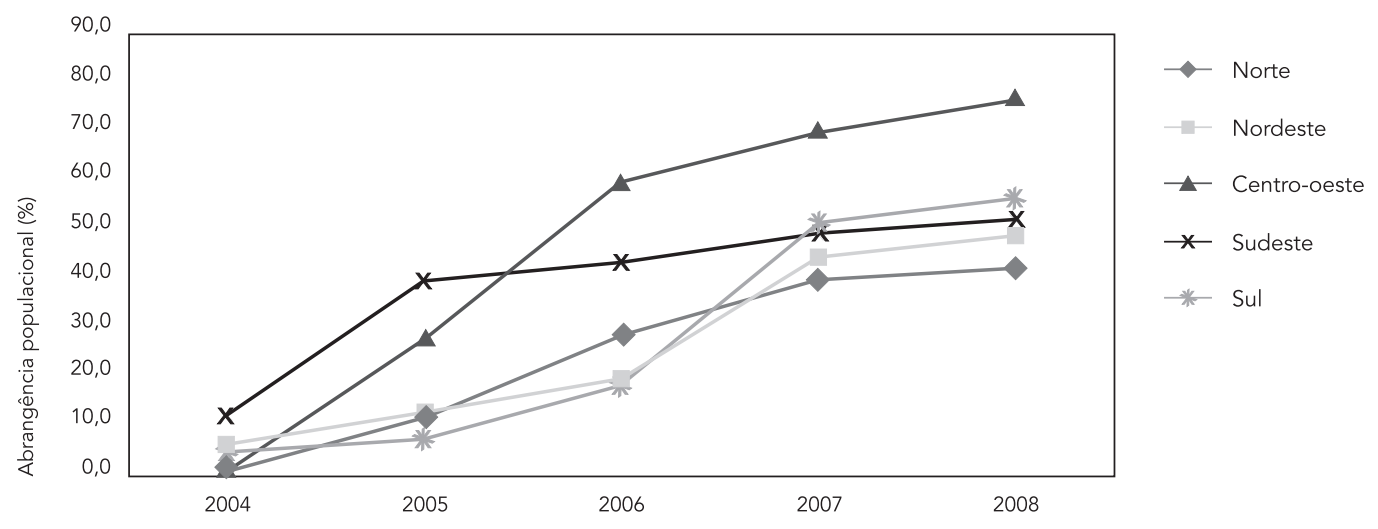

Fonte: dados dos SAMU fornecidos pela Coordenação Geral de Urgências e Emergências do Ministério da Saúde. Dados populacionais do Instituto Brasileiro de Geografia e Estatística.

saúde privados, admitindo a possibilidade de compra por pessoas atendidas no SUS.

Inicialmente, a estratégia se baseava na abertura de farmácias estatais, geridas diretamente pela Fundação Oswaldo Cruz ou por meio de parceria federal com estados e municípios (a maioria), cujo número em meados de 2008 ultrapassava 450 unidades no país. Em caso de parcerias, o Governo Federal oferece incentivo para instalação e manutenção das farmácias, enquanto estados e municípios contribuem com parte dos custos de manutenção e pagamento de pessoal. Tais farmácias disponibilizam para venda subsidiada pelo Ministério da Saúde mais de setenta medicamentos, incluindo aqueles voltados às doenças cardiovasculares, infecciosas, do sistema endócrino e anticoncepcionais orais. A venda é feita diretamente às pessoas com receita médica (de serviços públicos ou privados), e o usuário paga $10 \%$ do valor de referência e o Governo Federal arca com $90 \%$.

A partir de 2006, cresce uma segunda vertente do programa, representada pelo credenciamento de farmácias privadas da rede do comércio varejista em todo o país, cuja expansão se dá de forma bem mais acelerada nos anos subsequentes (Figura 3), alcançando mais de 6 mil estabelecimentos em 2008, com implicações importantes para o alcance nacional do programa e para a assistência farmacêutica.

A venda de medicamentos subsidiados pelo Governo Federal nessas farmácias privadas conveniadas é feita nos mesmos termos das far- mácias públicas (pagamento de $10 \%$ do valor de referência pelos usuários e $90 \%$ pelo Estado). $\mathrm{O}$ conjunto de medicamentos disponíveis é menor, incluindo drogas para controle da hipertensão arterial, diabetes e contracepção oral. A rápida expansão dos convênios fez, porém, que o número de atendimentos das farmácias privadas conveniadas ultrapassasse em pouco tempo os atendimentos das farmácias populares estatais, segundo dados do Ministério da Saúde. Ademais, foi proposta para os anos seguintes uma meta de aumento significativo dos convênios com farmácias privadas, bem como a expansão do conjunto de medicamentos cuja venda será subsidiada pelo Estado nessas farmácias 33.

Outra informação relevante se refere à distribuição regional das farmácias populares. A relação entre o número de farmácias e o tamanho da população varia entre as regiões do país de forma diferente para as duas vertentes do programa. A distribuição relativa à população das farmácias populares estatais, abertas mediante o estabelecimento de parcerias com municípios ou estados, vinha favorecendo as regiões Norte e Nordeste, de menor desenvolvimento econômico e com menor oferta de serviços de saúde. Já o peso de farmácias privadas conveniadas ao programa, que se expandem aceleradamente a partir de 2006, é mais expressivo nas regiões Sudeste e Sul, de maior desenvolvimento econômico, dada a maior oferta de serviços de saúde e força do comércio varejista de medicamentos nessas regiões (Figura 4). 
Evolução do número de farmácias estatais inauguradas e de farmácias privadas conveniadas ao programa Farmácia Popular. Brasil, 2004-2008.

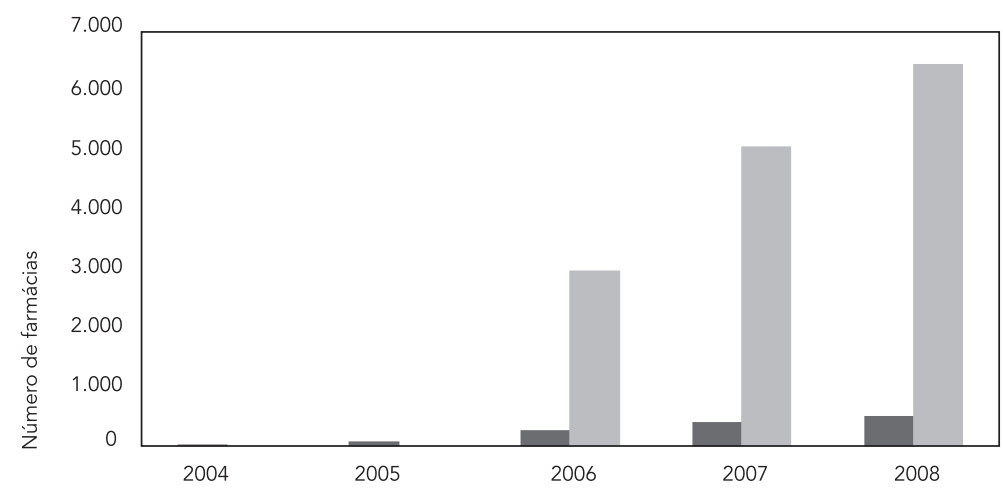

Farmácias estatais inauguradas

_ Farmácias privadas conveniadas

Fonte: dados da Sala de Situação do Ministério da Saúde.

Figura 4

Número de farmácias estatais e farmácias privadas conveniadas ao programa Farmácia Popular, por 1 milhão de habitantes, segundo regiões. Brasil, 2008.

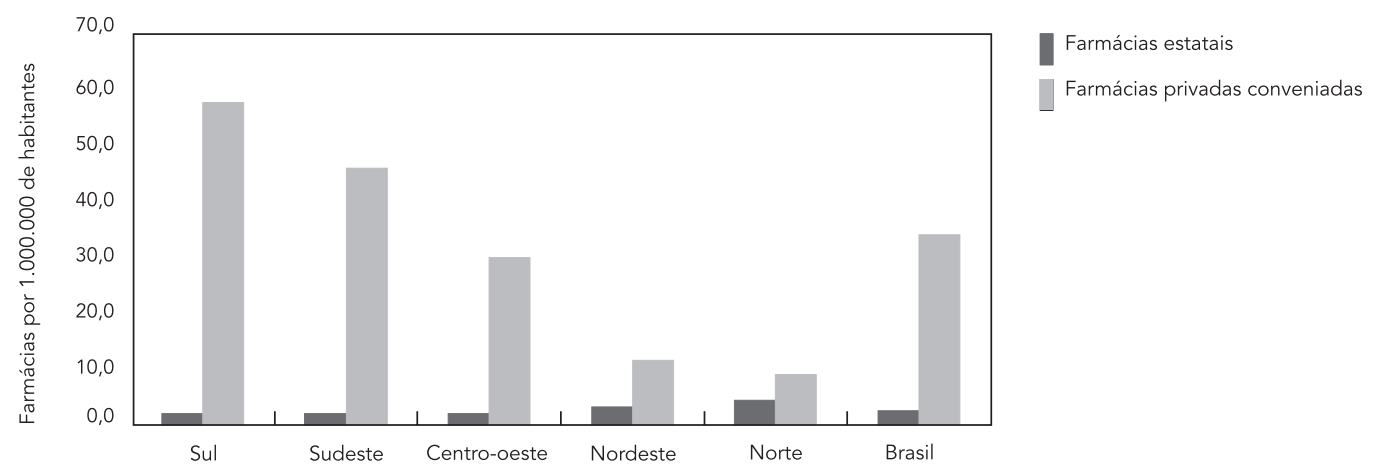

Fonte: dados do número de farmácias da Sala de Situação do Ministério da Saúde. Dados populacionais do Instituto Brasileiro de Geografia e Estatística.

Assim como os documentos oficiais, os dirigentes entrevistados ressaltaram que o programa convive com outras estratégias federais de assistência farmacêutica voltadas para a rede pública. De fato, entre 2004 e 2008, houve expansão de gastos federais direcionados a outros programas, embora o Farmácia Popular tenha apresentado o maior crescimento de gastos em termos relativos.

\section{Discussão}

As quatro políticas prioritárias no Governo Lula de 2003 a 2008 - ESF, Brasil Sorridente, SAMU e Farmácia Popular - representam estratégias voltadas para a solução de problemas relevantes do sistema de saúde brasileiro, respectivamente nas áreas de atenção primária à saúde, saúde bucal, atenção às urgências e assistência farmacêutica. 
A expansão do acesso nessas áreas é relevante para concretizar os princípios de universalidade e integralidade na saúde. Contudo, tais políticas apresentam diferenças importantes em termos da sua trajetória histórica, base político-institucional, forma de entrada na agenda federal e implicações para o SUS.

A permanência da ESF na agenda principal do Ministério da Saúde durante o Governo Lula, expressa um bom exemplo de "path dependen$c e$ ". Tal estratégia havia sido identificada como marco do governo anterior, quando foram definidas as suas diretrizes e regras de operação. No período estudado, a estratégia manteve destaque na agenda federal e se expandiu nos moldes definidos em meados dos anos 1990, apresentando somente mudanças incrementais. Algumas iniciativas de alterações mais substantivas no modelo entre 2003 e 2005 esbarraram nas opções políticas feitas anteriormente, nas regras institucionais vigentes e nas resistências de atores às mudanças, incluindo a própria burocracia federal da saúde e os dirigentes de outras esferas de governo 43 .

Dada a sua solidez e continuidade, pode-se dizer que a Saúde da Família tem-se configurado mais como uma política de Estado do que de um governo específico, ainda que as estratégias de expansão e de qualificação da política possam variar entre os governos federal, estaduais e municipais.

As outras políticas priorizadas na agenda federal da saúde - Brasil Sorridente, SAMU e Farmácia Popular - se mostraram atrativas como marcos diferenciais de governo. Essas estratégias contaram com investimentos em divulgação, objetivando garantir a sua associação com o Governo Federal, apesar da implementação descentralizada. Isso se traduziu na normatização detalhada de logomarcas, banners e cartazes a serem expostos nos serviços odontológicos, ambulâncias ou farmácias vinculadas às estratégias.

Outro ponto que as três apresentam em comum é trazer inovações em áreas em que o sistema de saúde apresentava fragilidades e havia lacunas de atuação federal, mas com diferenças em termos do processo político e entrada na agenda governamental.

O Brasil Sorridente logrou entrar na agenda federal no período de transição entre governos. Para Bartole 44, a habilidade dos profissionais que lutavam pelo fortalecimento da odontologia no SUS em associar a saúde bucal ao combate à fome, considerada a prioridade social do Governo Lula, teria favorecido a entrada do tema na agenda e o desenho de uma política abrangente para a área.
Cabe ressaltar que o Brasil Sorridente também expressa traços de "path - dependence", ao tomar como fundamento da política a constituição de equipes de saúde bucal, que já havia se iniciado durante o Governo FHC, em 2001, de forma análoga e vinculada à ESF. A existência prévia de regras institucionais e de equipes implantadas favoreceu a expansão acelerada das equipes nos anos subseqüentes, com continuidades em relação ao modelo anterior. Entretanto, uma inovação importante no período foi o investimento em serviços odontológicos especializados, fato que representou uma inflexão inédita na política nacional de saúde bucal e um avanço enquanto concretização do atendimento integral no sistema público de saúde.

A estratégia do SAMU também tem raízes anteriores ao Governo Lula, mas ganhou destaque inédito na agenda federal. Em que pese a importância do componente pré-hospitalar no atendimento às urgências, a rápida expansão dos SAMU expõe fragilidades referentes à formação dos profissionais que atuam nas ambulâncias e centrais reguladoras e à integração da rede de serviços de saúde 45 . Existem diferenças importantes entre os estados e municípios na implantação da política, relacionadas às experiências prévias e às características de organização da rede 45,46 .

Das quatro políticas prioritárias do período, a Farmácia Popular é a que compreende inovações mais substantivas, se considerada a trajetória da política de assistência farmacêutica no Brasil. O país foi pioneiro na adoção de uma relação de medicamentos essenciais para o sistema público (nos anos 1970) 47 e na provisão de medicamentos gratuitos para pessoas com AIDS (nos anos 1990) 48. Desde o início da implantação do SUS, observouse a persistência de problemas estruturais na assistência farmacêutica - fragmentação de programas, ineficiências na gestão, permeabilidade a interesses privados e dificuldades de acesso da população a medicamentos. Aconteceu, todavia, aumento da oferta de medicamentos no sistema público e avanços em áreas específicas. No início dos anos 2000, existiam mais de vinte programas federais de assistência farmacêutica, envolvendo medicamentos de atenção primária até os de alto custo, com dispensação gratuita 49 .

O programa Farmácia Popular, na prática, introduziu o copagamento Estado-usuário na aquisição de medicamentos no sistema de saúde brasileiro. Ainda que existam várias estratégias de assistência farmacêutica pública, parte da população atendida no SUS tem adquirido medicamentos por intermédio do programa. Um estudo valendo-se de dados oficiais apontou que, em 2007 , perto de $48 \%$ das pessoas que 
adquiriram medicamentos nas farmácias populares estatais do país fizeram-no com receitas emitidas por serviços públicos 50 . Outro estudo apontou uma menor disponibilidade de medicamentos selecionados em serviços públicos do que em farmácias populares, situação que pode explicar a busca do programa por usuários do SUS 51. Além do mais, dados da PNAD de 2008 sugerem a persistência de deficiências no acesso dos cidadãos a medicamentos nos serviços públicos de saúde 41 .

Ressalte-se que o copagamento de medicamentos, embora adotado por outros países, a princípio colide com as diretrizes legais do SUS, que prevêem o atendimento integral à saúde, inclusive na assistência farmacêutica. A introdução desse mecanismo em um contexto de acentuadas desigualdades de renda pode agravar a iniqüidade de acesso no sistema de saúde.

Outro problema se relaciona à indução de expansão de uma oferta farmacêutica que reitera o caráter mercantil da oferta de serviços de saúde, característica histórica do sistema de saúde brasileiro. Se no início o programa Farmácia Popular se baseava na abertura de farmácias estatais, a partir de 2006 sua aceleração implicou o estabelecimento de convênios com farmácias privadas para venda de medicamentos a preços subsidiados. No final de 2009, havia milhares de farmácias privadas credenciadas no país, com perspectiva de crescimento dessa vertente. Tal expansão requer novos mecanismos de regulação estatal sobre esse processo de dispensação de medicamentos, que ocorre fora das unidades de saúde.

É interessante ponderar que duas políticas analisadas - o Brasil Sorridente e o Farmácia Popular - se dirigem a áreas (assistência farmacêutica e odontologia) em que geralmente os gastos privados têm peso elevado, mesmo nos países com sistemas de saúde universais. Esses países respondem de forma diferenciada ao de- safio da atenção em tais áreas, muitas vezes com a adoção de mecanismos de restrição pelo lado da oferta ou da demanda. No caso brasileiro, enquanto o Brasil Sorridente enfatiza a expansão da oferta pública e gratuita de serviços, o programa Farmácia Popular induz a expansão da oferta privada e introduz o copagamento no sistema de saúde.

Em que pesem tais ressalvas, pesquisa qualitativa do governo em 2009 apontou o Farmácia Popular como o programa social federal com maior aprovação junto à população 52 .

Este artigo procurou identificar continuidades e mudanças na política de saúde no Governo Lula pela análise das prioridades da saúde, considerando os elementos de dependência da trajetória e a definição da agenda governamental. Cabe desenvolver estudos mais aprofundados de análise do processo e dos efeitos de cada uma das políticas. Um balanço mais completo da política deve considerar outros elementos além da agenda federal de prioridades, que não poderiam ser esgotados em um único artigo.

Ainda que as políticas nacionais de saúde no período expressem muitas continuidades, é importante mencionar que mudanças incrementais vêm sendo operadas. Ressaltem-se iniciativas de articulação da saúde com outros ministérios federais e com a agenda social do governo e um esforço recente do Ministério da Saúde de vincular o setor saúde ao debate sobre um novo modelo de desenvolvimento para o país.

Em meio à complexidade da política pública de saúde e às dificuldades de construção de um sistema nacional de caráter universal em um país heterogêneo e desigual, persistem desafios relacionados ao enfrentamento de problemas estruturais do sistema de saúde brasileiro. Entre eles, destacam-se a fragmentação das políticas, as limitações no financiamento, as distorções nas relações público-privadas e as marcantes desigualdades em saúde. 


\section{Resumo}

O artigo analisa as prioridades da política nacional da saúde no período de 2003 a 2008, correspondente ao Governo Lula. A pesquisa envolveu revisão bibliográfica, análise documental, análise de dados e entrevistas com dirigentes federais. Foram identificadas quatro prioridades na agenda federal da saúde: a Estratégia Saúde da Família, o Brasil Sorridente, os Serviços de Atendimento Móvel de Urgência e o programa Farmácia Popular. A primeira configura uma política de alta densidade institucional, iniciada no governo anterior, constituindo um exemplo de "dependência da trajetória". As demais foram adotadas como marcos de governo e trouxeram inovações em áreas em que havia fragilidades da atuação federal. As quatro políticas prioritárias analisadas se voltam para problemas relevantes do sistema de saúde brasileiro, porém apresentam diferenças quanto à sua trajetória, base de apoio e implicações para os princípios do Sistema Único de Saúde. Apesar de mudanças incrementais, observouse a predominância de elementos de continuidade na política nacional de saúde no período.

Política de Saúde; Agenda de Prioridades em Saúde; Sistema Único de Saúde; Governo Federal

\section{Referências}

1. Lima NT, Fonseca CMO, Hochman G. A saúde na construção do Estado Nacional no Brasil: reforma sanitária em perspectiva histórica. In: Lima NT, Gershman S, Edler FC, organizadores. Saúde e democracia: história e perspectivas do SUS. Rio de Janeiro: Editora Fiocruz, 2005. p. 27-58.

2. Escorel S. Reviravolta na saúde: origem e articulação do movimento sanitário. Rio de Janeiro: Editora Fiocruz; 1999.

3. Teixeira SF, organizadora. Reforma sanitária: em busca de uma teoria. São Paulo: Cortez Editora/ Rio de Janeiro: ABRASCO; 1989.

4. Paim JS. Reforma sanitária brasileira: contribuição para a compreensão e crítica. Salvador: EDUFBA, Rio de Janeiro: Editora Fiocruz; 2008.

5. Noronha JC, Levcovitz E. AIS, SUDS, SUS: os caminhos do direito à saúde. In: Guimarães R, Tavares R, organizadores. Saúde e sociedade no Brasil: anos 80. Rio de Janeiro: Relume-Dumará/ABRASCO/ Instituto de Medicina Social, Universidade do Estado do Rio de Janeiro; 1994. p. 73-111.

\section{Colaboradores}

C. V. Machado contribuiu com a concepção do artigo, realização da pesquisa, análise dos dados, redação e revisão final do artigo. T. W. F. Baptista participou da realização da pesquisa, análise dos dados, redação e revisão final do artigo. C. O. Nogueira participou da realização da pesquisa, sistematização e análise dos dados.

\section{Agradecimentos}

A pesquisa contou com apoio financeiro do Conselho Nacional de Desenvolvimento Científico e Tecnológico (CNPq), da Fundação Carlos Chagas de Amparo à Pesquisa do Estado do Rio de Janeiro (FAPERJ) e do Programa Estratégico de Apoio à Pesquisa em Saúde (PAPES/ Fiocruz). Durante a pesquisa, C. O. Nogueira foi bolsista Tecno-tecnologista da Fiocruz.
6. Sallum Jr. B. Crise, democratização e liberalização no Brasil. In: Sallum Jr. B, editor. Brasil e Argentina hoje: política e economia. Bauru: EDUSC; 2004. p. 47-77.

7. Noronha JC, Tavares LT. A política de saúde no Brasil nos anos 90. Ciênc Saúde Coletiva 2001; 6:44550.

8. Cordeiro H. Descentralização, universalidade e eqüidade nas reformas da saúde. Ciênc Saúde Coletiva 2001; 6:319-28.

9. Machado CV. Direito universal, política nacional: o papel do Ministério da Saúde na política de saúde brasileira de 1990 a 2002. Rio de Janeiro: Editora do Museu da República; 2007.

10. Baptista TWF. Políticas de saúde no pós-constituinte: um estudo da política implementada a partir da produção normativa dos poderes executivo e legislativo no Brasil [Dissertação de Mestrado]. Rio de Janeiro: Instituto de Medicina Social, Universidade do Estado do Rio de Janeiro; 2003. 
11. Instituto Brasileiro de Geografia e Estatística. Estatísticas da saúde: assistência médico-sanitária 2005. Rio de Janeiro: Instituto Brasileiro de Geografia e Estatística; 2006.

12. Vianna MLTW. Pyrrhic potatoes: comments on the institutional rules, macroeconomic constraints and innovation of the Brazilian social protection system in the 1990s and 2000s. Ciênc Saúde Coletiva 2008; 14:707-10.

13. Dain S. O financiamento público na perspectiva da política social. Economia e Sociedade 2001; 17:113-40.

14. Lima LD. Conexões entre o federalismo fiscal e o financiamento da política de saúde no Brasil. Ciênc Saúde Coletiva 2007; 12:511-22.

15. Souza C. Governos e sociedades locais em contextos de desigualdades e de descentralização. Ciênc Saúde Coletiva 2002; 42:593-634.

16. Viana ALA, Machado CV. Federative coordination and decentralization: Brazilian experience in health. Ciênc Saúde Coletiva 2008; 14:807-17.

17. Ribeiro P. Decentralization of governmental action in Brazil in the 90s: challenges of the politicalinstitutional environment. Ciênc Saúde Coletiva 2008; 14:819-27.

18. Ministério da Saúde/Organização Pan-Americana da Saúde. Observatório de Recursos Humanos no Brasil: estudos e análises. Brasília: Ministério da Saúde; 2004.

19. Gadelha CAG. Desenvolvimento, complexo industrial da saúde e política industrial. Rev Saúde Pública 2006; 40 Suppl:11-23.

20. Bahia L. The Brazilian health system between norms and facts: mitigated universalization and subsidized stratification. Ciênc Saúde Coletiva 2008; 14:753-62.

21. Andreazzi MFS, Kornis GEM. Transformações e desafios da atenção privada em saúde no Brasil nos anos 90. Physis (Rio J.) 2003; 13:157-91.

22. Draibe S. Brasil, 1980-2000: proteção e insegurança sociais em tempos difíceis. Campinas: Núcleo de Estudos de Políticas Públicas, Universidade Estadual de Campinas; 2005. (Caderno NEPP, 65).

23. Baptista TWF. Análise das portarias ministeriais da saúde e reflexões sobre a condução nacional da política de saúde. Cad Saúde Pública 2007; 23 : 615-26.

24. Machado CV. O modelo de intervenção do Ministério da Saúde brasileiro nos anos 90. Cad Saúde Pública 2007; 23:2113-26.

25. Figueras J, Musgrove P, Carrin G, Durán A. Retos para los sistemas sanitarios de Latinoamérica: ¿que puede aprenderse de la experiencia europea? Gac Sanit 2002; 16:5-17.

26. Soares S, Osório RG, Soares FV, Medeiros M, Zepeda E. Programas de transferência condicionada de renda no Brasil, Chile e México: impactos sobre a desigualdade. Brasília: Instituto de Pesquisa Econômica Aplicada; 2007. (Texto para Discussão, 1293).

27. Teixeira CF, Paim JS. A política de saúde no Governo Lula e a dialética do menos pior. Saúde Debate 2005; 29:268-83.
28. Bahia L. A saúde em banho-maria. In: Magalhães JPA, organizador. Os anos Lula: contribuições para um balanço crítico 2003-2010. Rio de Janeiro: Editora Garamond; 2010. p. 351-68.

29. Thelen K, Steinmo S. Historical institutionalism in comparative politics. In: Steinmo S, Thelen K, Longstrek F, editors. Structuring politics: historical institutionalism in comparative analysis. Cambridge: Cambridge University Press; 1992. p. 1-32.

30. Pierson P. Increasing returns, path dependence and the study of politics. Am Polit Sci Rev 2000; 94:251-67.

31. Kingdon JW. Agenda, alternatives and public policies. 2nd Ed. New York: HarperCollins College Publishers; 1995.

32. Ministério da Saúde. Portaria $n^{\circ}$. 2.607/GM de 10 de dezembro de 2004. Aprova o Plano Nacional de Saúde/PNS. Diário Oficial da União 2004; 13 dez.

33. Ministério da Saúde. Mais saúde - direito de todos: 2008-2011. 3a Ed. Brasília: Ministério da Saúde; 2009.

34. Ministério da Saúde. Política Nacional de Atenção Básica. Brasília: Ministério da Saúde; 2006.

35. Secretaria de Atenção à Saúde, Ministério da Saúde. Diretrizes da Política Nacional de Saúde Bucal. Brasília: Ministério da Saúde; 2004.

36. Secretaria de Atenção à Saúde, Ministério da Saúde. Regulação médica das urgências. Brasília: Ministério da Saúde; 2006.

37. Ministério da Saúde/Fundação Oswaldo Cruz. Programa Farmácia Popular do Brasil: manual básico. Brasília: Ministério da Saúde; 2005. (Série A - Manuais Técnicos).

38. Viana ALA, Dal Poz M. A reforma do sistema de saúde no Brasil e o Programa de Saúde da Família. Physis (Rio J.) 2005; 15 Suppl:225-64.

39. Machado CV. Prioridades da saúde no Brasil nos anos 1990: três políticas, muitas lições. Rev Panam Salud Pública 2006; 20:44-9.

40. Conill E. Ensaio histórico-conceitual sobre a Atenção Primária à Saúde: desafios para a organização de serviços básicos e da Estratégia Saúde da Família em centros urbanos no Brasil. Cad Saúde Pública 2008; 24 Suppl 1:S7-S27.

41. Viacava F. Acesso e uso dos serviços de saúde pelos brasileiros. Radis 2010; 96:12-9.

42. Narvai PC. Saúde bucal coletiva: caminhos da odontologia sanitária à bucalidade. Rev Saúde Pública 2006; 40(N Esp):141-7.

43. Castro ALB. A condução federal da política de atenção primária à saúde no Brasil: continuidades e mudanças no período de 2003 a 2008 [Dissertação de Mestrado]. Rio de Janeiro: Escola Nacional de Saúde Pública Sergio Arouca, Fundação Oswaldo Cruz; 2009.

44. Bartole M. Concepções e formulação de políticas e programas com enfoque da integralidade: o exemplo da Política Nacional de Saúde Bucal. In: Macau-Lopes MG, organizador. Saúde bucal coletiva: implementando idéias, concebendo integralidade. Rio de Janeiro: Editora Rubio; 2008. p. 161-73.

45. O'Dwyer G. A gestão da atenção às urgências e o protagonismo federal. Ciênc Saúde Coletiva 2010; 15:2395-404. 
46. Minayo MCS, Deslandes SF. Análise da implantação do sistema de atendimento pré-hospitalar móvel em cinco capitais brasileiras. Cad Saúde Pública 2008; 24:1877-86.

47. Pepe VLE, Castro CGSO, Luiza VL. A Relação Nacional de Medicamentos Essenciais: um instrumento da política nacional de medicamentos na garantia de acesso. In: Buss PM, Carvalheiro JR, Casas CPR, organizadores. Medicamentos no Brasil: inovação e acesso. Rio de Janeiro: Editora Fiocruz; 2008. p. 319-34.

48. Parker R. Construindo os alicerces para a resposta ao HIV/AIDS no Brasil: o desenvolvimento de políticas sobre o HIV/AIDS, 1982 - 1996. Divulg Saúde Debate 2003; 27:8-49.

49. Bermudez JAZ, Oliveira MA, Esher A, organizadores. Aceso a medicamentos: derecho fundamental, papel del Estado. Rio de Janeiro: Escola Nacional de Saúde Pública Sergio Arouca, Fundação Oswaldo Cruz; 2004.
50. Pinto CBS, Castro CGSO, Costa NR. Quem acessa o Programa Farmácia Popular do Brasil? Aspectos do fornecimento público de medicamentos. Ciênc Saúde Coletiva; 2008. http://www.abrasco.org.br/ cienciaesaudecoletiva/artigos/artigo_int.php?id_ artigo $=3056$ (acessado em Jun/2009).

51. Pinto CBS, Miranda ES, Emmerick CM, Costa NR, Castro CGSO. Preços e disponibilidade de medicamentos no Programa Farmácia Popular do Brasil. Rev Saúde Pública 2010; 44:611-9.

52. Formenti L. Em ano eleitoral, governo "turbina" Farmácia Popular. O Estado de S. Paulo 2010; 7 mai.

Recebido em 05/Mai/2010

Versão final reapresentada em 17/Nov/2010

Aprovado em 03/Jan/2011 